\title{
Frequent development of leptomeningeal carcinomatosis in patients with peritoneal dissemination of gastric cancer
}

\author{
Shigenobu Emoto $\cdot$ Hironori Ishigami • \\ Hironori Yamaguchi · Hiroharu Yamashita • \\ Shoichi Kaisaki · Joji Kitayama
}

Received: 21 December 2010/Accepted: 17 May 2011/Published online: 11 June 2011

(C) The International Gastric Cancer Association and The Japanese Gastric Cancer Association 2011

\begin{abstract}
We detected 7 cases of leptomeningeal carcinomatosis in 126 patients with peritoneal dissemination of gastric cancer who received combined systemic and intraperitoneal chemotherapy. Leptomeningeal carcinomatosis was diagnosed 79-1540 days after the diagnosis of the primary gastric cancer. Patients presenting with various neurological symptoms were diagnosed by cerebrospinal fluid (CSF) cytology and radiological imaging. Irradiation to the whole brain and spine was performed in 4 patients, and provided palliation and increased survival for 1 patient. Intrathecal chemotherapy and drainage of CSF was performed in 1 patient each, but produced no significant clinical benefit in either of them. Survival after the diagnosis of leptomeningeal carcinomatosis was between 3 and 155 days. As patients with peritoneal dissemination of gastric cancer are living longer because of improved chemotherapy, clinicians must recognize the possibility of leptomeningeal carcinomatosis when patients complain of neurological symptoms.
\end{abstract}

Keywords Leptomeningeal carcinomatosis .

Gastric cancer · Peritoneal dissemination .

Intraperitoneal chemotherapy

\section{Introduction}

Leptomeningeal metastasis (LMC) is diagnosed in 1-5\% of patients with solid tumors [1]. Prognosis for patients with

S. Emoto $(\bowtie) \cdot H$. Ishigami · H. Yamaguchi - H. Yamashita ·

S. Kaisaki · J. Kitayama

Department of Surgical Oncology, The University of Tokyo,

7-3-1 Hongo, Bunkyo-ku, Tokyo 113-8655, Japan

e-mail: semoto-tky@umin.ac.jp
LMC is poor and they experience severe symptoms such as headache, nausea, and vomiting. Adenocarcinoma is the most common form of LMC, and the breast, lung, and skin are the most common primary sites [1,2]. Despite recent advances in systemic chemotherapy, the prognoses for patients with LMC from gastric cancer remain poor, with a median length of survival of 4-6 weeks $[1,3]$.

LMC is thought to be relatively rare in gastric cancer, with a frequency of $0.16-0.69 \%$ in all gastric cancer patients [4-8]. Peritoneal metastasis is the most frequent and life-threatening mode of metastasis and recurrence in patients with gastric cancer. Recently, intraperitoneal chemotherapy with a taxane has been shown to effectively treat gastric cancer with peritoneal dissemination $[9,10]$. For patients with peritoneal metastasis, we recently developed a regimen of repeated intraperitoneal administrations of paclitaxel combined with systemic chemotherapy; this dramatically prolonged patient survival [11]. Of the 126 patients we treated for gastric cancer with peritoneal dissemination, 7 patients had LMC. Here, we report the detailed clinical aspects of these 7 patients, because we believe that this high rate of occurrence needs to be highlighted.

\section{Case reports}

From February 2005 to October 2010, 126 consecutive patients were diagnosed with gastric cancer and peritoneal metastases, and $7(5.6 \%)$ of these patients were diagnosed with LMC. The disease characteristics and outcomes of these 7 patients are summarized in Table 1. The patients' ages ranged from 36 to 86 years (median 52 years). Six patients were initially diagnosed with peritoneal dissemination or malignant ascites; the seventh patient was initially diagnosed with $\mathrm{T} 4 \mathrm{aN} 1$ gastric cancer and underwent 


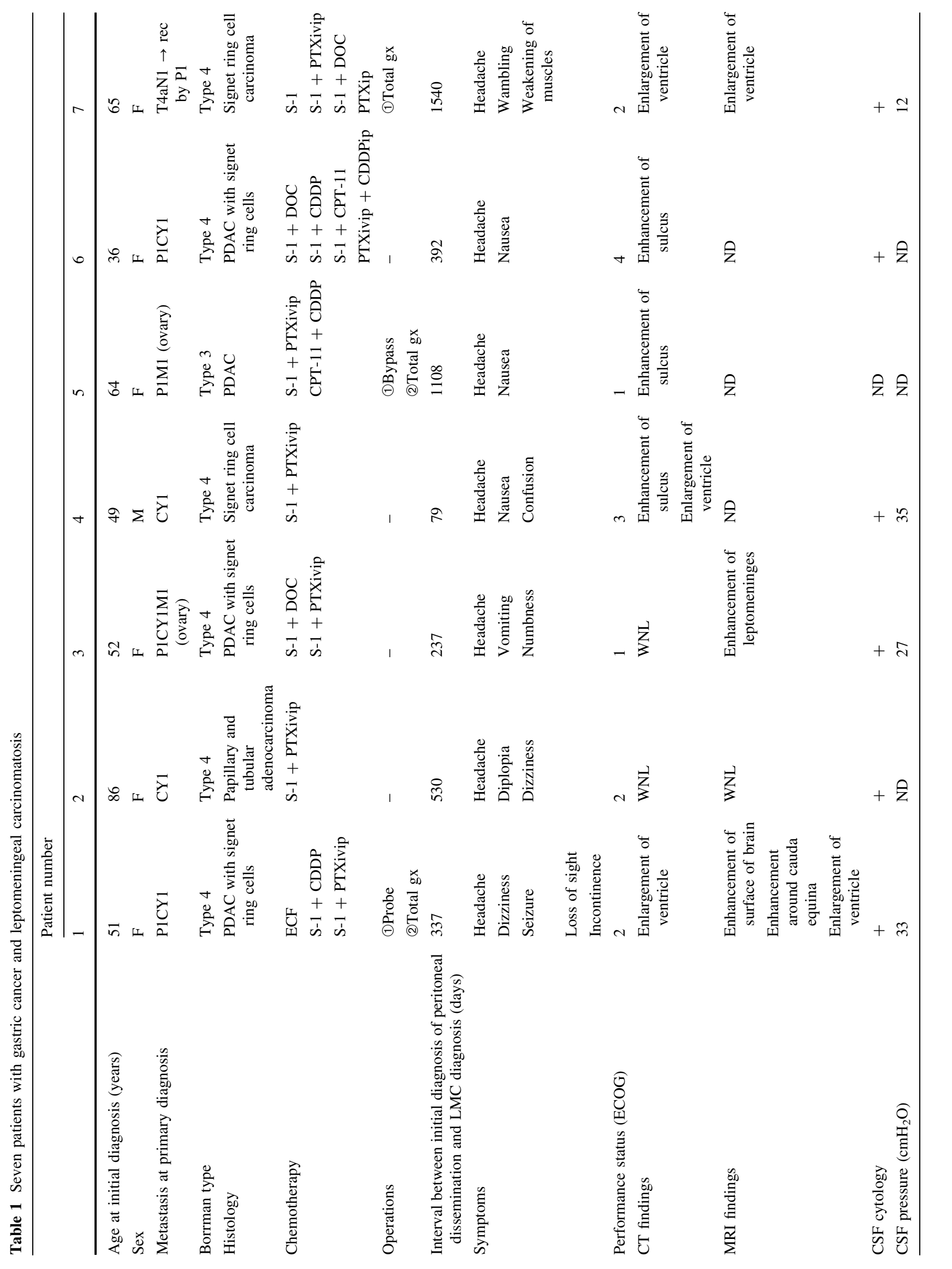




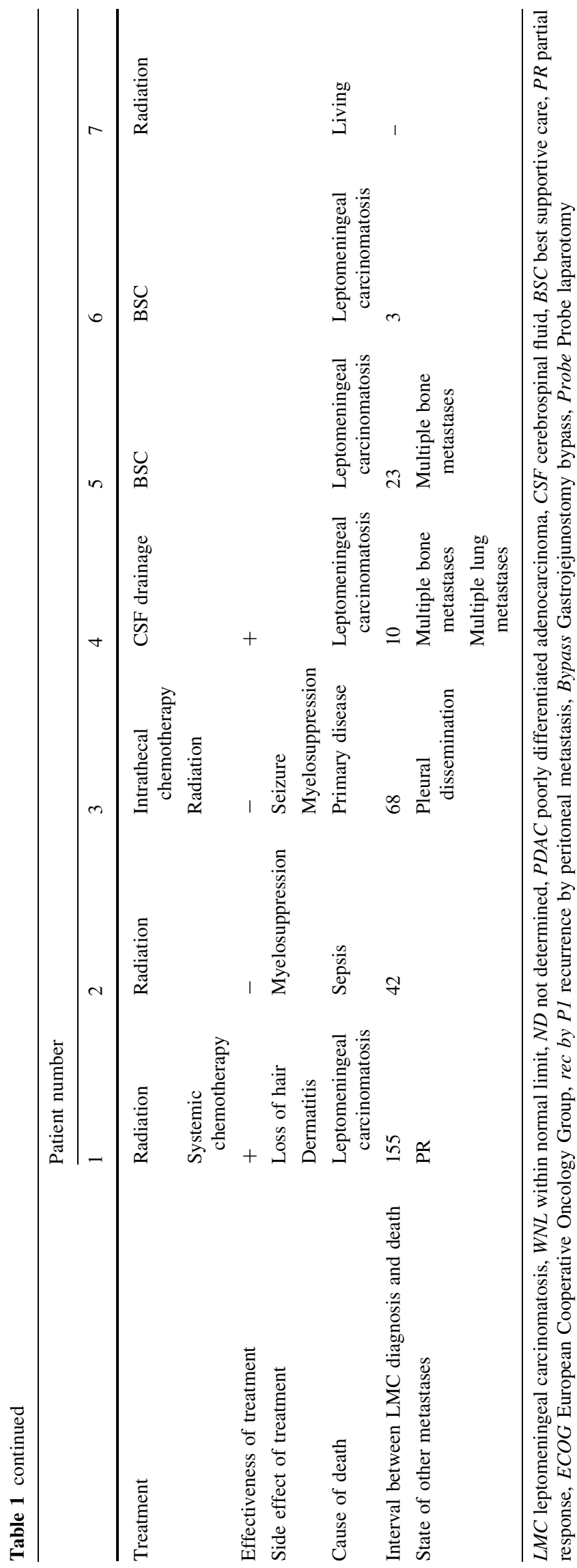

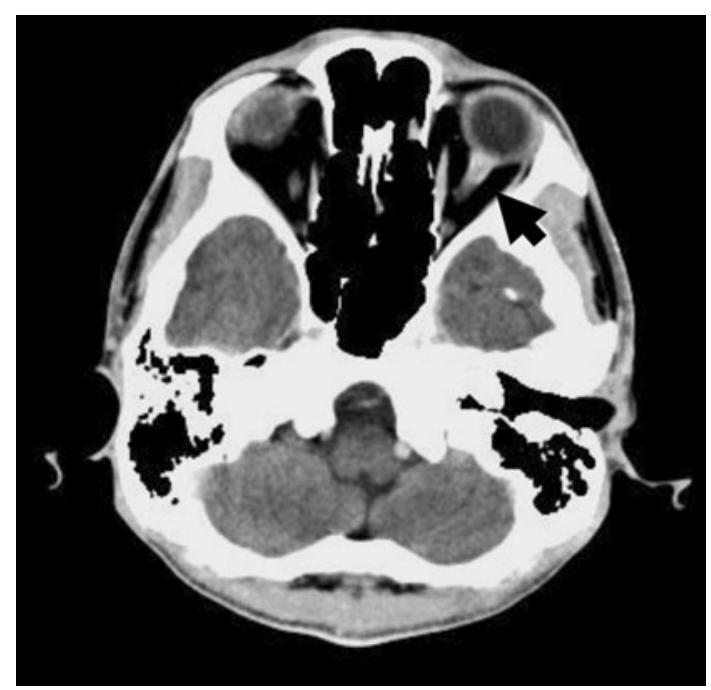

Fig. 1 Enhancement along the left optic nerve (arrow) on contrastenhanced computed tomography (CT) (Case 4)

a total gastrectomy, developing a peritoneal recurrence 5.5 years after the surgery. The macroscopic type of gastric cancer was Borrman type 4 in 6 patients and type 3 in 1 patient. Histological examination showed that 6 of the 7 patients had poorly differentiated adenocarcinoma or signet ring cell carcinoma and that 1 had tubular and papillary adenocarcinoma. All patients received combined chemotherapy including intraperitoneal paclitaxel. Two patients had total gastrectomies after complete remission of the peritoneal metastases. The interval between the initial diagnosis of peritoneal metastasis and that of LMC ranged from 79 to 1540 days (median 392).

In our 7 patients, a variety of symptoms were observed, such as headache, nausea or dizziness, seizure, decreased vision, diplopia, confusion, and limb numbness. All the patients complained of persistent headaches, which were thought to have been caused by elevated intracranial pressure. However, none of the patients showed signs of meningeal irritation such as Kernig's sign or neck rigidity.

Contrast-enhanced brain computed tomography (CT) was performed for all patients. Enhancement along the cerebral sulcus, reported to be specific to LMC, was detected in 3 patients, all of whom died within 1 month. Significant enlargement of the cerebral ventricles was identified in 2 patients, suggesting abnormal flow of cerebrospinal fluid (CSF) and elevated CSF pressure. The sulcus and fissure of 1 patient was narrowed, suggesting progression of cerebral edema. In another patient, enhancement along the optic nerve was observed (Fig. 1). The cancer had possibly spread along the nerve's sheath, indicating the importance of careful inspection of all sites in the image, given that the leptomeninges cover the entire brain and spine. 
For 4 patients, we performed gadolinium $(\mathrm{Gd})$-enhanced magnetic resonance imaging (MRI), because there was no specific evidence of LMC on the enhanced CT scans, though LMC was strongly suspected. In 2 patients, positive enhancement was detected along the leptomeninges (Fig. 2), and in 1 patient, abnormal enhancement was detected around a nerve root of the cauda equina. No abnormality was identified in the fourth patient.

Examination of lumbar puncture and CSF cytology revealed adenocarcinoma in 6 patients. CSF pressure was examined in 4 patients, 3 of whom showed elevated pressure $\left(27-35 \mathrm{cmH}_{2} \mathrm{O}\right)$.

One patient was treated with intrathecal (IT) administration of methotrexate (MTX). In this patient, we injected $15 \mathrm{mg}$ of MTX intrathecally once a week, repeating this treatment 3 times. However, the patient developed systemic seizures after each MTX administration; therefore, the IT chemotherapy was discontinued. Whole-brain irradiation was performed in 4 patients; 1 patient reported dramatic relief from headaches and nausea, enabling the oral intake of food and continuation of systemic chemotherapy until her death 155 days after the LMC diagnosis. For the other 2 patients, treatment was limited to best supportive care (BSC) because of their impaired general status. For 1 patient, CSF was drained through lumbar puncture to relieve headaches caused by elevated intracranial pressure. However, the pain relief only lasted for several hours, necessitating repeated drainage.

Six patients died between 3 and 155 days after the diagnosis of LMC, with the median survival length being 33 days; 1 patient was alive for 30 days after the diagnosis of LMC. Four of these 6 patients died of sudden apnea, which appeared to be the most frequent cause of death in
LMC. The other 2 patients died of sepsis or multiple organ failure. An autopsy was performed in 1 patient (Case 1 in Table 1). She died of sudden cessation of respiration and we suspected cerebral hernia. In the autopsy examination, the cerebrum was swollen, with opacified leptomeninges, but cerebral hernia was not detected. Histologically, the carcinomatosis was spread throughout the leptomeninges and the IT space of the entire brain and cord, and minute areas of invasion to the cerebral parenchyma were observed on the surface of the medulla oblongata and the pons.

\section{Discussion}

We found that 7 of $126(5.6 \%)$ patients with peritoneal metastasis of gastric cancer developed LMC during the course of chemotherapy; this frequency is much higher than that previously reported for all types of gastric cancer $[1,2,6]$. Our results suggest that LMC is undiagnosed in many cases because the patient's general condition is severely impaired. However, the average length of survival for patients with peritoneal metastasis of gastric cancer is approximately 1 year $[12,13]$. Given that, in our series, the median interval between the diagnosis of peritoneal metastasis and the diagnosis of LMC was 392 days, it is possible that most patients with peritoneal dissemination die before developing LMC.

Our patients' clinical manifestations were generally consistent with those previously reported [1, 2, 5, 7], as was their median length of survival (33 days) [1, 3]. Cytology of the CSF is the gold standard for LMC cancer diagnosis. In our series, all 6 patients who underwent the
Fig. 2 Enhancement along the leptomeninges (arrows) on Gdenhanced magnetic resonance imaging (MRI) (Case 3)
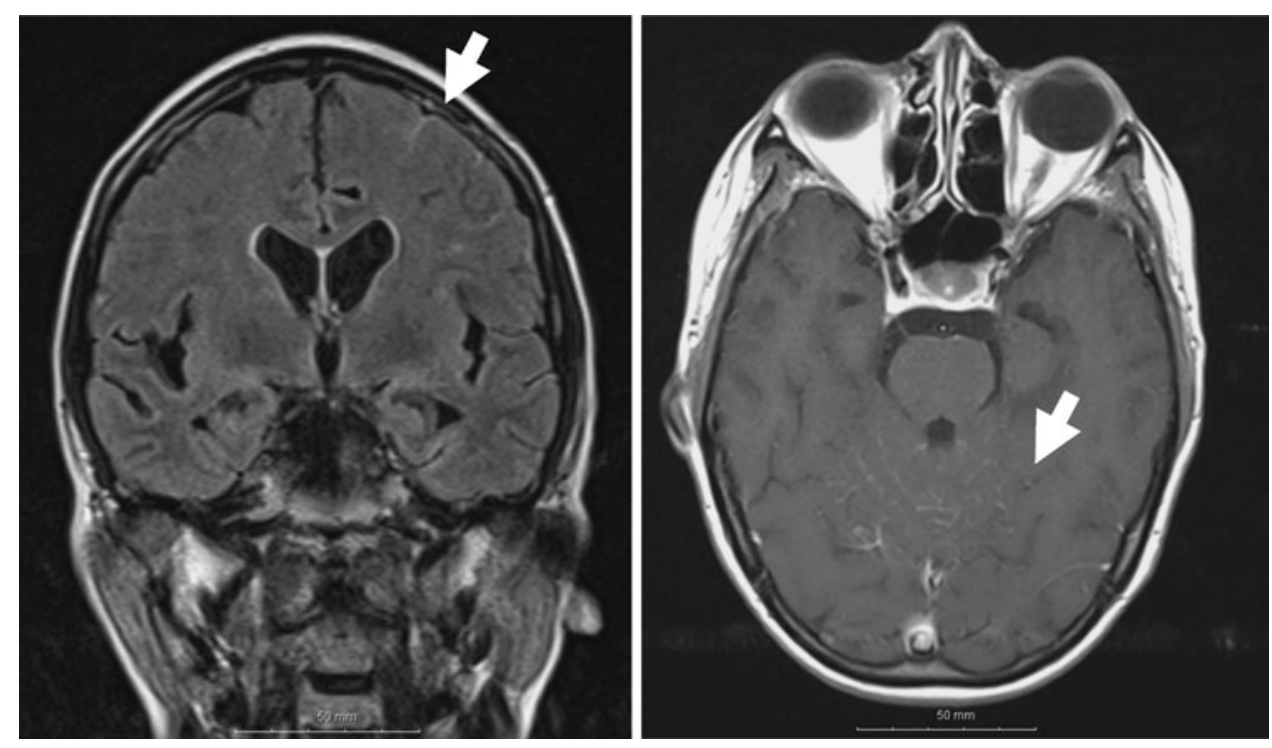
CSF cytology examination were shown to have malignant cells in the initial lumbar puncture, although false-negatives have often been reported [3]. The central nervous symptoms of LMC are sometimes misdiagnosed as the toxicity of chemotherapeutic agents. However, given our results, we recommend CSF cytology for patients with neurological symptoms that occur during treatment for peritoneal dissemination.

Recent studies suggest that the standard tool for imaging LMC is Gd-enhanced MRI [5, 14, 15] and that a combination of enhanced MRI and CSF cytology be used for accurate diagnosis of LMC [3]. MRI enhancement along the leptomeninges is specific to meningitis. Spinal cord involvement and fluid abnormalities can also be detected by MRI. Although MRI is generally regarded as superior to CT for diagnosing LMC, contrast-enhanced CT scans are often used initially, as they are more readily available. In our study, we detected abnormalities from contrast-enhanced CT images in 5 of the 7 patients. For 3 of these patients, we found positive enhancement along the cerebral sulcus, and the length of survival of these patients was extremely short (3-23 days). Given the successful detection of abnormalities in most of our patients and the high risk of sudden death for some patients, we suggest that CT scans are clinically useful.

IT chemotherapy using MTX, cytosine arabinoside (Ara-C), and thiotepa is considered to be one option for the treatment of LMC [1, 3, 16], though the efficacy of this regimen is still unclear. Kim and colleagues [17] have reported that a combination of MTX and Ara-C is more effective than the administration of MTX alone. Previous reports indicate that patients receiving IT chemotherapy live longer than those receiving $\mathrm{BSC}[16,18]$, and Oh et al. [6] report that negative conversion of CSF cytology by IT chemotherapy increases survival. However, these results may be affected by the accuracy of CSF cytology, as well as by differences in the general condition of the selected patients. In our study, we performed IT chemotherapy using MTX in 1 patient, and this treatment was ineffective. Waki and colleagues [19] reported that in patients with poor prognostic factors such as poor performance status or MRI-proven LMC, palliative therapy may be the most suitable treatment strategy. Large-scale clinical studies are required for standardizing IT chemotherapy.

Whole-brain radiation is often performed for LMC patients to palliate their symptoms, decrease bulky disease, and correct CSF flow abnormalities. In our study, 4 patients received radiation treatment, and this treatment increased the length of survival for 1 patient. CSF drainage is also performed to relieve the symptoms of elevated intracranial pressure $[1,20]$. In our experience, CSF drainage by lumbar puncture temporarily alleviated headaches; thus, the use of an Ommaya reservoir $[21,22]$ may be useful to avoid repeated puncture. IT chemotherapy, whole-brain radiation, or other palliative treatment should be chosen on a case-by-case basis, tailored to the clinical status of each patient.

In summary, LMC often develops in patients with peritoneal dissemination of gastric cancer. The outcome for patients with LMC is poor, and many die suddenly of apnea. As patients with peritoneal dissemination of gastric cancer are living longer because of improved chemotherapy, clinicians must recognize the possibility of LMC when such patients complain of neurological symptoms such as headache, nausea, and dizziness.

\section{References}

1. Chamberlain MC. Leptomeningeal metastasis. Curr Opin Oncol. 2010;22:627-35.

2. Wasserstrom WR, Glass JP, Posner JB. Diagnosis and treatment of leptomeningeal metastases from solid tumors: experience with 90 patients. Cancer. 1982;49:759-72.

3. Groves MD. New strategies in the management of leptomeningeal metastases. Arch Neurol. 2010;67:305-12.

4. Lee JL, Kang YK, Kim TW, Chang HM, Lee GW, Ryu MH, et al. Leptomeningeal carcinomatosis in gastric cancer. J Neurooncol. 2004;66:167-74.

5. Lisenko Y, Kumar AJ, Yao J, Ajani J, Ho L. Leptomeningeal carcinomatosis originating from gastric cancer: report of eight cases and review of the literature. Am J Clin Oncol. 2003;26:165-70

6. Oh SY, Lee SJ, Lee J, Lee S, Kim SH, Kwon HC, et al. Gastric leptomeningeal carcinomatosis: multi-center retrospective analysis of 54 cases. World J Gastroenterol. 2009;15:5086-90.

7. Ohno T, Yokoyama Y, Aihara R, Mochiki E, Asao T, Kuwano H. Sudden bilateral sensorineural hearing loss as the presenting symptom of meningeal carcinomatosis of gastric cancer: report of a case. Surg Today. 2010;40:561-5.

8. Raj KP, Sanati H, Mehta RS, Zell JA. Need for a new treatment strategy: leptomeningeal carcinomatosis from gastric cancer. Anticancer Drugs. 2009;20:301-4.

9. Kodera $\mathrm{Y}$, Ito $\mathrm{Y}$, Ito $\mathrm{S}$, Ohashi N, Mochizuki Y, Yamamura Y, et al. Intraperitoneal paclitaxel: a possible impact of regional delivery for prevention of peritoneal carcinomatosis in patients with gastric carcinoma. Hepatogastroenterology. 2007;54:960-3.

10. Tamura S, Miki H, Okada K, Miyake T, Yoshimura M, Suzuki R, et al. Pilot study of intraperitoneal administration of paclitaxel and oral S-1 for patients with peritoneal metastasis due to advanced gastric cancer. Int J Clin Oncol. 2008;13:536-40.

11. Ishigami H, Kitayama J, Kaisaki S, Hidemura A, Kato M, Otani $\mathrm{K}$, et al. Phase II study of weekly intravenous and intraperitoneal paclitaxel combined with S-1 for advanced gastric cancer with peritoneal metastasis. Ann Oncol. 2010;21:67-70.

12. Glehen O, Mohamed F, Gilly FN. Peritoneal carcinomatosis from digestive tract cancer: new management by cytoreductive surgery and intraperitoneal chemohyperthermia. Lancet Oncol. 2004;5:219-28.

13. Imazawa M, Kojima $T$, Boku N, Onozawa $Y$, Hironaka S, Fukutomi A, et al. Efficacy of sequential methotrexate and 5-fluorouracil (MTX/5FU) in improving oral intake in patients with advanced gastric cancer with severe peritoneal dissemination. Gastric Cancer. 2009;12:153-7.

14. Clarke JL, Perez HR, Jacks LM, Panageas KS, Deangelis LM. Leptomeningeal metastases in the MRI era. Neurology. 2010;74:1449-54. 
15. Straathof CS, de Bruin HG, Dippel DW, Vecht CJ. The diagnostic accuracy of magnetic resonance imaging and cerebrospinal fluid cytology in leptomeningeal metastasis. J Neurol. 1999;246:810-4.

16. Beauchesne P. Intrathecal chemotherapy for treatment of leptomeningeal dissemination of metastatic tumours. Lancet Oncol. 2010;11:871-9.

17. Kim DY, Lee KW, Yun T, Park SR, Jung JY, Kim DW, et al. Comparison of intrathecal chemotherapy for leptomeningeal carcinomatosis of a solid tumor: methotrexate alone versus methotrexate in combination with cytosine arabinoside and hydrocortisone. Jpn J Clin Oncol. 2003;33:608-12.

18. Orlando L, Curigliano G, Colleoni M, Fazio N, Nole F, Martinelli $\mathrm{G}$, et al. Intrathecal chemotherapy in carcinomatous meningitis from breast cancer. Anticancer Res. 2002;22:3057-9.
19. Waki F, Ando M, Takashima A, Yonemori K, Nokihara H, Miyake $\mathrm{M}$, et al. Prognostic factors and clinical outcomes in patients with leptomeningeal metastasis from solid tumors. J Neurooncol. 2009;93:205-12.

20. Omuro AM, Lallana EC, Bilsky MH, DeAngelis LM. Ventriculoperitoneal shunt in patients with leptomeningeal metastasis. Neurology. 2005;64:1625-7.

21. Takeda T, Saitoh M, Takeda S. Solitary cystic brain metastasis of small-cell lung carcinoma controlled by a stereotactically inserted Ommaya reservoir. Am J Med Sci. 2009;337:215-7.

22. Yoshida S, Morii K. Intrathecal chemotherapy for patients with meningeal carcinomatosis. Surg Neurol. 2005;63:52-5. (discussion 5). 